\title{
Generalized Weyl's theorem for algebraically quasi-paranormal operators
}

\author{
II Ju An and Young Min Han
}

* Correspondence:

ymhan2004@khu.ac.kr

Department Of Mathematics,

College Of Sciences, Kyung Hee

University, Seoul 130-701, Republic

Of Korea

\begin{abstract}
Let $T$ or $T^{*}$ be an algebraically quasi-paranormal operator acting on a Hilbert space. We prove: (i) generalized Weyl's theorem holds for $f(T)$ for every $f \in H(\sigma(T))$; (ii) generalized a-Browder's theorem holds for $f(S)$ for every $S \prec T$ and $f \in H(\sigma(S))$; (iii) the spectral mapping theorem holds for the B-Weyl spectrum of $T$. Moreover, we show that if $T$ is an algebraically quasi-paranormal operator, then $T+F$ satisfies generalized Weyl's theorem for every algebraic operator $F$ which commutes with $T$. Mathematics Subject Classification (2010): Primary 47A10, 47A53; Secondary 47B20.
\end{abstract}

Keywords: algebraically quasi-paranormal operator, generalized Weyl's theorem, single valued extension property

\section{Introduction}

Throughout this article, we assume that $\mathcal{H}$ is an infinite dimensional separable Hilbert space. Let $B(\mathcal{H})$ and $B_{0}(\mathcal{H})$ denote, respectively, the algebra of bounded linear operators and the ideal of compact operators acting on $\mathcal{H}$. If $T \in B(\mathcal{H})$ we shall write $N(T)$ and $R(T)$ for the null space and range of $T$. Also, let $\alpha(T):=\operatorname{dim} N(T), \beta(T):=\operatorname{dim} N\left(T^{*}\right)$, and let $\sigma$ $(T), \sigma_{a}(T), \sigma_{p}(T), \pi(T), E(T)$ denote the spectrum, approximate point spectrum, point spectrum of $T$, the set of poles of the resolvent of $T$, the set of all eigenvalues of $T$ which are isolated in $\sigma(T)$, respectively. An operator $T \in B(\mathcal{H})$ is called upper semi-Fredholm if it has closed range and finite dimensional null space and is called lower semi-Fredholm if it has closed range and its range has finite co-dimension. If $T \in B(\mathcal{H})$ is either upper or lower semi-Fredholm, then $T$ is called semi-Fredholm, and index of a semi-Fredholm operator $T \in B(\mathcal{H})$ is defined by

$$
i(T):=\alpha(T)-\beta(T) .
$$

If both $\alpha(T)$ and $\beta(T)$ are finite, then $T$ is called Fredholm. $T \in B(\mathcal{H})$ is called Weyl if it is Fredholm of index zero. For $T \in B(\mathcal{H})$ and a nonnegative integer $n$ define $T_{n}$ to be the restriction of $T$ to $R\left(T^{n}\right)$ viewed as a map from $R\left(T^{n}\right)$ into $R\left(T^{n}\right)$ (in particular $T_{0}=T$ ). If for some integer $n$ the range $R\left(T^{n}\right)$ is closed and $T_{n}$ is upper (resp. lower) semi-Fredholm, then $T$ is called upper (resp. lower) semi-B-Fredholm. Moreover, if $T_{n}$ is Fredholm, then $T$ is called B-Fredholm. $T$ is called semi-B-Fredholm if it is upper or lower semi-BFredholm. Let $T$ be semi- $B$-Fredholm and let $d$ be the degree of stable iteration of $T$. It follows from [1, Proposition 2.1] that $T_{m}$ is semi-Fredholm and $i\left(T_{m}\right)=i\left(T_{d}\right)$ for each $m$

(c) 2012 An and Han; licensee Springer. This is an Open Access article distributed under the terms of the Creative Commons Attribution License (http://creativecommons.org/licenses/by/2.0), which permits unrestricted use, distribution, and reproduction in any medium, provided the original work is properly cited. 
$\geq d$. This enables us to define the index of semi-B-Fredholm $T$ as the index of semiFredholm $T_{d}$. Let $B F(\mathcal{H})$ be the class of all $B$-Fredholm operators. In [2], they studied this class of operators and they proved [2, Theorem 2.7] that an operator $T \in B(\mathcal{H})$ is $B$-Fredholm if and only if $T=T_{1} \oplus T_{2}$, where $T_{1}$ is Fredholm and $T_{2}$ is nilpotent. It appears that the concept of Drazin invertibility plays an important role for the class of $B$-Fredholm operators. Let $\mathcal{A}$ be a unital algebra. We say that an element $x \in \mathcal{A}$ is Drazin invertible of degree $k$ if there exists an element $a \in \mathcal{A}$ such that

$$
x^{k} a x=x^{k}, a x a=a, \text { and } x a=a x .
$$

Let $a \in \mathcal{A}$. Then the Drazin spectrum is defined by

$$
\sigma_{D}(a):=\{\lambda \in \mathbb{C}: a-\lambda \text { is not Drazin invertible }\} .
$$

For $T \in B(\mathcal{H})$, the smallest nonnegative integer $p$ such that $N\left(T^{p}\right)=N\left(T p+^{1}\right)$ is called the ascent of $T$ and denoted by $p(T)$. If no such integer exists, we set $p(T)=\infty$. The smallest nonnegative integer $q$ such that $R\left(T^{q}\right)=R\left(T^{q+1}\right)$ is called the descent of $T$ and denoted by $q(T)$. If no such integer exists, we set $q(T)=\infty$. It is well known that $T$ is Drazin invertible if and only if it has finite ascent and descent, which is also equivalent to the fact that

$$
T=T_{1} \oplus T_{2} \text {, where } T_{1} \text { is invertible and } T_{2} \text { is nilpotent. }
$$

An operator $T \in B(\mathcal{H})$ is called $B$-Weyl if it is $B$-Fredholm of index 0 . The $B$-Fredholm spectrum $\sigma_{B F}(T)$ and $B$-Weyl spectrum $\sigma_{B W}(T)$ of $T$ are defined by

$$
\begin{aligned}
& \sigma_{B F}(T):=\{\lambda \in \mathbb{C}: T-\lambda \text { is not } B-\text { Fredholm }\}, \\
& \sigma_{B W}(T):=\{\lambda \in \mathbb{C}: T-\lambda \text { is not } B-\text { Weyl }\} .
\end{aligned}
$$

Now, we consider the following sets:

$$
\begin{aligned}
& B F_{+}(\mathcal{H}):=\{T \in B(\mathcal{H}): T \text { is upper semi }-B-\text { Ferdholm }\} \\
& B F_{+}^{-}(\mathcal{H}):=\left\{T \in B(\mathcal{H}): T \in B F_{+}(\mathcal{H}) \text { and } i(T) \leq 0\right\} \\
& L D(\mathcal{H}):=\left\{T \in B(\mathcal{H}): p(T)<\infty \text { and } R\left(T^{p(T)+1}\right) \text { is closed }\right\} .
\end{aligned}
$$

By definition,

$$
\sigma_{\text {Bea }}(T):=\left\{\lambda \in \mathbb{C}: T-\lambda \notin B F_{+}^{-}(\mathcal{H})\right\},
$$

is the upper semi- $B$-essential approximate point spectrum and

$$
\sigma_{L D}(T):=\{\lambda \in \mathbb{C}: T-\lambda \notin L D(\mathcal{H})\}
$$

is the left Drazin spectrum. It is well known that

$$
\sigma_{B e a}(T) \subseteq \sigma_{L D}(T)=\sigma_{B e a}(T) \cup \operatorname{acc} \sigma_{a}(T) \subseteq \sigma_{D}(T),
$$

where we write acc $K$ for the accumulation points of $K \subseteq \mathbb{C}$. If we write iso $K:=K \backslash$ acc $K$ then we let

$$
\begin{aligned}
p_{0}^{a}(T) & :=\left\{\lambda \in \sigma_{\alpha}(T): T-\lambda \in L D(\mathcal{H})\right\}, \\
\pi_{0}^{a}(T) & :=\left\{\lambda \in \text { iso } \sigma_{a}(T): \lambda \in \sigma_{p}(T)\right\} .
\end{aligned}
$$


We say that an operator $T$ has the single valued extension property at $\lambda$ (abbreviated SVEP at $\lambda$ ) if for every open set $U$ containing $\lambda$ the only analytic function $f: U \rightarrow \mathcal{H}$ which satisfies the equation

$$
(T-\lambda) f(\lambda)=0
$$

is the constant function $f \equiv 0$ on $U$. $T$ has SVEP if $T$ has SVEP at every point $\lambda \in \mathbb{C}$.

Definition 1.1. Let $T \in B(\mathcal{H})$.

(1) Generalized Weyl's theorem holds for $T$ (in symbols, $T \in g \mathcal{W}$ ) if

$$
\sigma(T) \backslash \sigma_{B W}(T)=E(T) .
$$

(2) Generalized Browder's theorem holds for $T$ (in symbols, $T \in g \mathcal{B}$ ) if

$\sigma(T) \backslash \sigma_{B W}(T)=\pi(T)$.

(3) Generalized a-Weyl's theorem holds for $T$ (in symbols, $T \in g a \mathcal{W}$ ) if

$$
\sigma_{a}(T) \backslash \sigma_{B e a}(T)=\pi_{0}^{a}(T) .
$$

(4) Generalized a-Browder's theorem holds for $T$ (in symbols, $T \in g a \mathcal{B}$ ) if

$$
\sigma_{a}(T) \backslash \sigma_{B e a}(T)=p_{0}^{a}(T) .
$$

It is known ([3]) that the following set inclusions hold:

$$
\begin{array}{cc}
g a-\text { Weyl's theorem } & \Rightarrow g a-\text { Browder's theorem } \\
\Downarrow & \Downarrow \\
g-\text { Weyl's theorem } \Rightarrow g-\text { Browder's theorem }
\end{array}
$$

Recently, Han and Na introduced a new operator class which contains the classes of paranormal operators and quasi-class $A$ operators [4]. In [5], it was shown that generalized Weyl's theorem holds for algebraically paranormal operators. In this article, we extend this result to algebraically quasi-paranormal operators using the local spectral theory

\section{Generalized Weyl's theorem for algebraically quasi-paranormal operators}

Definition 2.1. (1) An operator $T \in B(\mathcal{H})$ is said to be class $A$ if

$$
|T|^{2} \leq\left|T^{2}\right| .
$$

(2) $T$ is called a quasi-class $A$ operator if

$$
T^{*}|T|^{2} T \leq T^{*}\left|T^{2}\right| T .
$$

(3) An operator $T \in B(\mathcal{H})$ is said to be paranormal if

$$
\|T x\|^{2} \leq\left\|T^{2} x\right\|\|x\| \quad \text { for all } x \in \mathcal{H} .
$$

Recently, we introduced a new operator class which is a common generalization of paranormal operators and quasi-class $A$ operators [4].

Definition 2.2. An operator $T \in B(\mathcal{H})$ is called quasi-paranormal if

$$
\left\|T^{2} x\right\|^{2} \leq\left\|T^{3} x\right\|\|T x\| \quad \text { for all } x \in \mathcal{H} .
$$


We say that $T \in B(\mathcal{H})$ is an algebraically quasi-paranormal operator if there exists a non-constant complex polynomial $h$ such that $h(T)$ is quasi-paranormal.

In general, the following implications hold:

class $A \Rightarrow$ quasi-class $A \Rightarrow$ quasi-paranormal;

paranormal $\Rightarrow$ quasi-paranormal $\Rightarrow$ algebraically quasi-paranormal.

In [4], it was observed that there are examples which are quasi-paranormal but not paranormal, as well as quasi-paranormal but not quasi-class $A$. We give a more simple example which is quasi-paranormal but not quasi-class $A$. To construct this example we recall the following lemma in [4].

Lemma 2.3. An operator $T \in B(\mathcal{H})$ is quasi-paranormal if and only if

$$
T^{*}\left(T^{2 *} T^{2}-2 \lambda T^{*} T+\lambda^{2}\right) T \geq 0 \text { for all } \lambda>0 .
$$

Example 2.4. $T=\left(\begin{array}{ll}I & 0 \\ I & 0\end{array}\right) \in B\left(\ell_{2} \oplus \ell_{2}\right)$. Then it is quasi-paranormal but not quasiclass

A.

Proof. Since $T^{*}=\left(\begin{array}{cc}I & I \\ 0 & 0\end{array}\right),\left|T^{2}\right|=\sqrt{\left(T^{*}\right)^{2} T^{2}}=\sqrt{\left(\begin{array}{cc}I & I \\ 0 & 0\end{array}\right)^{2}\left(\begin{array}{ll}I & 0 \\ I & 0\end{array}\right)^{2}=\left(\begin{array}{cc}\sqrt{2} I & 0 \\ 0 & 0\end{array}\right)}$

Therefore $T^{*}\left|T^{2}\right| T=\left(\begin{array}{cc}I & I \\ 0 & 0\end{array}\right)\left(\begin{array}{cc}\sqrt{2} I & 0 \\ 0 & 0\end{array}\right)\left(\begin{array}{ll}I & 0 \\ I & 0\end{array}\right)=\left(\begin{array}{cc}\sqrt{2} I & 0 \\ 0 & 0\end{array}\right)$

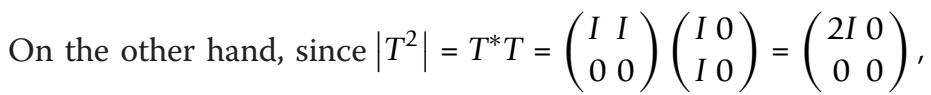

$T^{*}\left|T^{2}\right| T=\left(\begin{array}{ll}I & I \\ 0 & 0\end{array}\right)\left(\begin{array}{cc}2 I & 0 \\ 0 & 0\end{array}\right)\left(\begin{array}{ll}I & 0 \\ I & 0\end{array}\right)=\left(\begin{array}{ll}2 I & 0 \\ 0 & 0\end{array}\right)$. Hence $T$ is not quasi-class $A$.

However, since

$$
T^{2 *} T^{2}-2 \lambda T^{*} T+\lambda^{2}=\left(\begin{array}{cc}
\left(2-4 \lambda+\lambda^{2}\right) I & 0 \\
0 & \lambda^{2} I
\end{array}\right)
$$

we have

$$
T^{*}\left(T^{2 *} T^{2}-2 \lambda T^{*} T+\lambda^{2}\right) T=\left(\begin{array}{cr}
2(1-\lambda)^{2} I & 0 \\
0 & 0
\end{array}\right) \geq 0
$$

for all $\lambda>0$. Therefore $T$ is quasi-paranormal.

The following example provides an operator which is algebraically quasi-paranormal but not quasi-paranormal.

Example 2.5 Let $T=\left(\begin{array}{ll}I & 0 \\ I & I\end{array}\right) \in B\left(\ell_{2} \oplus \ell_{2}\right)$. Then it is algebraically quasi-paranormal but not quasi-paranormal.

Proof. Since $T^{*}=\left(\begin{array}{ll}I & I \\ 0 & I\end{array}\right)$, we have

$$
T^{2 *} T^{2}-2 \lambda T^{*} T+\lambda^{2}=\left(\begin{array}{cc}
\left(\lambda^{2}-4 \lambda+5\right) I & (-2 \lambda+2) I \\
(-2 \lambda+2) I & \left(\lambda^{2}-2 \lambda+1\right) I
\end{array}\right) .
$$


Therefore

$$
T^{*}\left(T^{2 *} T^{2}-2 \lambda T^{*} T+\lambda^{2}\right) T=\left(\begin{array}{cc}
\left(2 \lambda^{2}-10 \lambda+10\right) I & \left(\lambda^{2}-4 \lambda+3\right) I \\
\left(\lambda^{2}-4 \lambda+3\right) I & \left(\lambda^{2}-2 \lambda+1\right) I
\end{array}\right) .
$$

Since $\left(2 \lambda^{2}-10 \lambda+10\right) I$ is not a positive operator for $\lambda=2$, $T^{*}\left(T^{2 *} T^{2}-2 \lambda T^{*} T+\lambda^{2}\right) T \nsucceq 0$ for $\lambda>0$. Therefore $T$ is not quasi-paranormal. On the other hand, consider the complex polynomial $h(z)=(z-1)^{2}$. Then $h(T)=0$, and hence $T$ is algebraically quasi-paranormal.

The following facts follow from the above definition and some well known facts about quasi-paranormal operators [4]:

(i) If $T \in B(\mathcal{H})$ is algebraically quasi-paranormal, then so is $T-\lambda$ for each $\lambda \in \mathbb{C}$.

(ii) If $T \in B(\mathcal{H})$ is algebraically quasi-paranormal and $\mathcal{M}$ is a closed $T$-invariant subspace

of $\mathcal{H}$, then $T \mid \mathcal{M}$ is algebraically quasi-paranormal.

(iii) If $T$ is algebraically quasi-paranormal, then $T$ has SVEP.

(iv) Suppose $T$ does not have dense range. Then we have:

$T$ is quasi-paranormal $\Leftrightarrow T=\left(\begin{array}{ll}A & B \\ 0 & 0\end{array}\right)$ on $\mathcal{H}=\overline{T(\mathcal{H})} \oplus N\left(T^{*}\right)$,

where $A=T \mid \overline{T(\mathcal{H})}$ is paranormal.

An operator $T \in B(\mathcal{H})$ is called isoloid if iso $\sigma(T) \subseteq \sigma_{p}(T)$ and an operator $T \in B(\mathcal{H})$ is called polaroid if iso $\sigma(T) \subseteq \pi(T)$.

In general, the following implications hold:

$$
T \text { polaroid } \Rightarrow T \text { isoloid. }
$$

However, each converse is not true. Consider the following example: let $T \in B\left(\ell_{2}\right)$ be defined by

$$
T\left(x_{1}, x_{2}, x_{3}, \ldots\right)=\left(\frac{1}{2} x_{2}, \frac{1}{3} x_{3}, \ldots\right) .
$$

Then $T$ is a compact quasinilpotent operator with $\alpha(T)=1$, and so $T$ is isoloid. However, since $q(T)=\infty, T$ is not polaroid.

An important subspace in local spectral theory is the quasi-nilpotent part of $T$ defined by

$$
H_{0}(T):=\left\{x \in \mathcal{H}: \lim _{n \rightarrow \infty}\left\|T^{n} x\right\| \frac{1}{n}=0\right\}
$$

If $T \in B(\mathcal{H})$, then the analytic core $K(T)$ is the set of all $x \in \mathcal{H}$ such that there exists a constant $c>0$ and a sequence of elements $x_{n} \in \mathcal{H}$ such that $x_{0}=x, T x_{n}=x_{n-1}$, and $\left\|x_{n}\right\| \leq c^{n}\|x\|$ for all $n \in \mathbb{N}$, see [6] for information on $K(T)$.

Let $\mathcal{P}(\mathcal{H})$ denotes the class of all operators for which there exists $p:=p(\lambda) \in \mathbb{N}$ for which 


$$
H_{0}(T-\lambda)=N(T-\lambda)^{p} \text { for all } \lambda \in \mathbb{C},
$$

and $\mathcal{P}_{1}(\mathcal{H})$ denotes the class of all operators for which there exists $p:=p(\lambda) \in \mathbb{N}$ for which

$$
H_{0}(T-\lambda)=N(T-\lambda)^{p} \text { for all } \lambda \in E(T) .
$$

Evidently, $\mathcal{P}(\mathcal{H}) \subseteq \mathcal{P}_{1}(\mathcal{H})$. Now we give a characterization of $\mathcal{P}_{1}(\mathcal{H})$

Theorem 2.6. $T \in \mathcal{P}_{1}(\mathcal{H})$ if and only if $\pi(T)=E(T)$.

Proof. Suppose $T \in \mathcal{P}_{1}(\mathcal{H})$ and let $\lambda \in E(T)$. Then there exists $p \in \mathbb{N}$ such that $H_{0}(T$ $\lambda)=N(T-\lambda)^{p}$. Since $\lambda$ is an isolated point of $\sigma(T)$, it follows from [6, Theorem 3.74] that

$$
\mathcal{H}=H_{0}(T-\lambda) \oplus K(T-\lambda)=N(T-\lambda)^{p} \oplus K(T-\lambda) .
$$

Therefore, we have

$$
(T-\lambda)^{p}(\mathcal{H})=(T-\lambda)^{p}(K(T-\lambda))=K(T-\lambda),
$$

and hence $\mathcal{H}=N(T-\lambda)^{p} \oplus(T-\lambda)^{p}(\mathcal{H})$, which implies, by [6, Theorem 3.6], that $p$ $(T-\lambda)=q(T-\lambda) \leq p$. But $\alpha(T-\lambda)>0$, hence $\lambda\lfloor\pi(T)$. Therefore $E(T) \subseteq \pi(T)$. Since the opposite inclusion holds for every operator $T$, we then conclude that $\pi(T)=E(T)$. Conversely, suppose $\pi(T)=E(T)$. Let $\lambda\lfloor E(T)$. Then $p:=p(T-\lambda)=q(T-\lambda)<\infty$. By [6, Theorem 3.74], $H_{0}(T-\lambda)=N(T-\lambda)^{p}$. Therefore $T \in \mathcal{P}_{1}(\mathcal{H})$. $\square$

From Theorem 2.6, we can give a simple example which belongs to $\mathcal{P}_{1}(\mathcal{H})$ but not $\mathcal{P}(\mathcal{H})$. Let $U$ be the unilateral shift on $\ell_{2}$ and let $T=U^{*}$. Then $T$ does not have SVEP at 0 , and so $H_{0}(T)$ is not closed. Therefore $T \notin \mathcal{P}(\mathcal{H})$. However, since $\sigma(T)=\overline{\mathbb{D}}, \pi(T)=E(T)=\emptyset$, where $\mathbb{D}$ is an open unit disk in $\mathbb{C}$. Hence $T \in \mathcal{P}_{1}(\mathcal{H})$ by Theorem 2.6.

Before we state our main theorem (Theorem 2.9) in this section, we need some preliminary results.

Lemma 2.7. Let $T \in B(\mathcal{H})$ be a quasinilpotent algebraically quasi-paranormal operator. Then $T$ is nilpotent.

Proof. We first assume that $T$ is quasi-paranormal. We consider two cases:

Case I: Suppose $T$ has dense range. Then clearly, it is paranormal. Therefore $T$ is nilpotent by [7, Lemma 2.2].

Case II: Suppose $T$ does not have dense range. Then we can represent $T$ as the upper triangular matrix

$$
T=\left(\begin{array}{ll}
A & B \\
0 & 0
\end{array}\right) \text { on } \mathcal{H}=\overline{T(\mathcal{H})} \oplus N\left(T^{*}\right),
$$

where $A:=T \mid \overline{T(\mathcal{H})}$ is an paranormal operator. Since $T$ is quasinilpotent, $\sigma(T)=\{0\}$. But $\sigma(T)=\sigma(A) \cup\{0\}$, hence $\sigma(A)=\{0\}$. Since $A$ is paranormal, $A=0$ and therefore $T$ is nilpotent. Thus if $T$ is a quasinilpotent quasi-paranormal operator, then it is nilpotent. Now, we suppose $T$ is algebraically quasi-paranormal. Then there exists a nonconstant polynomial $p$ such that $p(T)$ is quasi-paranormal. If $p(T)$ has dense range, then $p(T)$ is paranormal. So $T$ is algebraically paranormal, and hence $T$ is nilpotent by [7, Lemma 2.2]. If $p(T)$ does not have dense range, we can represent $p(T)$ as the upper triangular matrix 


$$
p(T)=\left(\begin{array}{ll}
C & D \\
0 & 0
\end{array}\right) \text { on } \mathcal{H}=\overline{p(T)(\mathcal{H})} \oplus N\left(p(T)^{*}\right),
$$

where $C:=p(T) \mid \overline{p(T)(\mathcal{H})}$ is paranormal. Since $T$ is quasinilpotent, $\sigma(p(T))=p(\sigma(T))$ $=\{p(0)\}$. But $\sigma(p(T))=\sigma(C) \cup\{0\}$ by $[8$, Corollary 8], hence $\sigma(C) \cup\{0\}=\{p(0)\}$. So $p(0)=$ 0 , and hence $p(T)$ is quasinilpotent. Since $p(T)$ is quasi-paranormal, by the previous argument $p(T)$ is nilpotent. On the other hand, since $p(0)=0, p(z)=c z^{m}\left(z-\lambda_{1}\right)(z$ $\left.\lambda_{2}\right) \ldots\left(z-\lambda_{n}\right)$ for some natural number $m$. Therefore $p(T)=c T^{m}(T-\lambda 1)\left(T-\lambda_{2}\right) \ldots(T$ $\left.-\lambda_{n}\right)$. Since $p(T)$ is nilpotent and $T-\lambda_{i}$ is invertible for every $\lambda_{i} \neq 0, T$ is nilpotent. This completes the proof. $\square$

Theorem 2.8. Let $T \in B(\mathcal{H})$ be algebraically quasi-paranormal. Then $T \in \mathcal{P}_{1}(\mathcal{H})$.

Proof. Suppose $T$ is algebraically quasi-paranormal. Then $h(T)$ is a quasi-paranormal operator for some nonconstant complex polynomial $h$. Let $\lambda \in E(T)$. Then $\lambda$ is an isolated point of $\sigma(T)$ and $\alpha(T-\lambda)>0$. Using the spectral projection $P:=\frac{1}{2 \pi i} \int_{\partial D}(\mu-T)^{-1} d \mu$, where $D$ is a closed disk of center $\lambda$ which contains no other points of $\sigma(T)$, we can represent $T$ as the direct sum

$$
T=\left(\begin{array}{cc}
T_{1} & 0 \\
0 & T_{2}
\end{array}\right), \text { where } \sigma\left(T_{1}\right)=\{\lambda\} \text { and } \sigma\left(T_{2}\right)=\sigma(T) \backslash\{\lambda\} .
$$

Since $T_{1}$ is algebraically quasi-paranormal, so is $T_{1}-\lambda$. But $\sigma\left(T_{1}-\lambda\right)=\{0\}$, it follows from Lemma 2.7 that $T_{1}-\lambda$ is nilpotent. Therefore $T_{1}-\lambda$ has finite ascent and descent. On the other hand, since $T_{2}-\lambda$ is invertible, clearly it has finite ascent and descent. Therefore $\lambda$ is a pole of the resolvent of $T$, and hence $\lambda \in \pi(T)$. Hence $E(T) \subseteq$ $\pi(T)$. Since $\pi(T) \subseteq E(T)$ holds for any operator $T$, we have $\pi(T)=E(T)$. It follows from Theorem 2.6 that $T \in \mathcal{P}_{1}(\mathcal{H})$. $\square$

We now show that generalized Weyl's theorem holds for algebraically quasi-paranormal operators. In the following theorem, recall that $H(\sigma(T))$ is the space of functions analytic in an open neighborhood of $\sigma(T)$.

Theorem 2.9. Suppose that $T$ or $T^{*}$ is an algebraically quasi-paranormal operator. Then $f(T) \in g \mathcal{W}$ for each $f \in H(\sigma(T))$.

Proof. Suppose $T$ is algebraically quasi-paranormal. We first show that $T \in g \mathcal{W}$. Suppose that $\lambda \in \sigma(T) \backslash \sigma_{B W}(T)$. Then $T-\lambda$ is $B$-Weyl but not invertible. It follows from [9, Lemma 4.1] that we can represent $T-\lambda$ as the direct sum

$$
T-\lambda=\left(\begin{array}{cc}
T_{1} & 0 \\
0 & T_{2}
\end{array}\right), \text { where } T_{1} \text { is Weyl and } T_{2} \text { is nilpotent. }
$$

Since $T$ is algebraically quasi-paranormal, it has SVEP. So $T_{1}$ and $T_{2}$ have both finite ascent. But $T_{1}$ is Weyl, hence $T_{1}$ has finite descent. Therefore $T-\lambda$ has finite ascent and descent, and so $\lambda \in E(T)$. Conversely, suppose that $\lambda \in E(T)$. Since $T$ is algebraically quasi-paranormal, it follows from Theorem 2.8 that $T \in \mathcal{P}_{1}(\mathcal{H})$. Since $\pi(T)=E$ (T) by Theorem 2.6, $\lambda \in E(T)$. Therefore $T-\lambda$ has finite ascent and descent, and so we can represent $T-\lambda$ as the direct sum

$$
T-\lambda=\left(\begin{array}{cc}
T_{1} & 0 \\
0 & T_{2}
\end{array}\right), \text { where } T_{1} \text { is invertible and } T_{2} \text { is nilpotent. }
$$


Therefore $T-\lambda$ is $B$-Weyl, and so $\lambda \in \sigma(T) \backslash \sigma_{B W}(T)$. Thus $\sigma(T) \backslash \sigma_{B W}(T)=E(T)$, and hence $T \in g \mathcal{W}$.

Next, we claim that $\sigma_{B W}(f(T))=f\left(\sigma_{B W}(T)\right)$ for each $f \in H(\sigma(T))$. Since $T \in g \mathcal{W}, T \in g \mathcal{B}$. It follows from [5, Theorem 2.1] that $\sigma_{B W}(T)=\sigma_{D}(T)$. Since $T$ is algebraically quasi-paranormal, $f(T)$ has SVEP for each $f \in H(\sigma(T))$. Hence $f(T) \in g \mathcal{B}$ by [5, Theorem 2.9], and so $\sigma_{B W}(f(T))=\sigma_{D}(f(T))$. Therefore we have

$$
\sigma_{B W}(f(T))=\sigma_{D}(f(T))=f\left(\sigma_{D}(T)\right)=f\left(\sigma_{B W}(T)\right) .
$$

Since $T$ is algebraically quasi-paranormal, it follows from the proof of Theorem 2.8 that it is isoloid. Hence for any $f \in H(\sigma(T))$ we have

$$
\sigma(f(T)) \backslash E(f(T))=f(\sigma(T) \backslash E(T)) .
$$

Since $T \in g \mathcal{W}$, we have

$$
\sigma(f(T)) \backslash E(f(T))=f(\sigma(T) \backslash E(T))=f\left(\sigma_{B W}(T)\right)=\sigma_{B W}(f(T)),
$$

which implies that $f(T) \in g \mathcal{W}$.

Now suppose that $T^{*}$ is algebraically quasi-paranormal. We first show that $T \in g \mathcal{W}$. Let $\lambda \in \sigma(T) \backslash \sigma_{B W}(T)$. Observe that $\sigma\left(T^{*}\right)=\overline{\sigma(T)}$ and $\sigma_{B W}\left(T^{*}\right)=\overline{\sigma_{B W}(T)}$. So $\bar{\lambda} \in \sigma\left(T^{*}\right) \backslash \sigma_{B W}\left(T^{*}\right)$, and so $\bar{\lambda} \in E\left(T^{*}\right)$ because $T^{*} \in g \mathcal{W}$. Since $T^{*}$ is algebraically quasi-paranormal, it follows from Theorem 2.8 that $\bar{\lambda} \in \pi\left(T^{*}\right)$. Hence $T-\lambda$ has finite ascent and descent, and so $\lambda \in E(T)$. Conversely, suppose $\lambda \in E(T)$. Then $\lambda$ is an isolated point of $\sigma(T)$ and $\alpha(T-\lambda)>0$. Since $\sigma\left(T^{*}\right)=\overline{\sigma(T)}, \bar{\lambda}$ is an isolated point of $\sigma$ $\left(T^{*}\right)$. Since $T^{*}$ is isoloid, $\bar{\lambda} \in E\left(T^{*}\right)$. But $E\left(T^{*}\right)=\pi\left(T^{*}\right)$ by Theorem 2.8 , hence we have $T-\lambda$ has finite ascent and descent. Therefore we can represent $T-\lambda$ as the direct sum

$$
T-\lambda=\left(\begin{array}{cc}
T_{1} & 0 \\
0 & T_{2}
\end{array}\right), \text { where } T_{1} \text { is invertible and } T_{2} \text { is nilpotent. }
$$

Therefore $T-\lambda$ is $B$-Weyl, and so $\lambda \in \sigma(T) \backslash \sigma_{B W}(T)$. Thus $\sigma(T) \backslash \sigma_{B W}(T)=E(T)$, and hence $T \in g \mathcal{W}$. If $T^{*}$ is algebraically quasi-paranormal then $T$ is isoloid. It follows from the first part of the proof that $f(T) \in g \mathcal{W}$. This completes the proof. $\square$

From the proof of Theorem 2.9 and [10, Theorem 3.4], we obtain the following useful consequence.

Corollary 2.10. Suppose $T$ or $T^{*}$ is algebraically quasi-paranormal. Then

$$
\sigma_{B W}(f(T))=f\left(\sigma_{B W}(T)\right) \quad \text { for every } f \in H(\sigma(T)) .
$$

An operator $X \in B(\mathcal{H})$ is called a quasiaffinity if it has trivial kernel and dense range. $S \in B(\mathcal{H})$ is said to be a quasiaffine transform of $T \in B(\mathcal{H})$ (notation: $S \prec T$ ) if there is a quasiaffinity $X \in B(\mathcal{H})$ such that $X S=T X$. If both $S \prec T$ and $T \prec S$, then we say that $S$ and $T$ are quasisimilar.

Corollary 2.11. Suppose $T$ is algebraically quasi-paranormal and $S \prec T$. Then $f(S) \in g a \mathcal{B}$ for each $f \in H(\sigma(S))$.

Proof. Suppose $T$ is algebraically quasi-paranormal. Then $T$ has SVEP. Since $S<T, f$ $(S)$ has SVEP by [7, Lemma 3.1]. It follows from [11, Theorem 3.3.6] that $f(S)$ has SVEP. Therefore $f(S) \in g a \mathcal{B}$ by [12, Corollary 2.5]. $\square$ 


\section{Generalized Weyl's theorem for perturbations of algebraically quasi- paranormal operators}

An operator $T$ is said to be algebraic if there exists a nontrivial polynomial $h$ such that $h(T)=0$. From the spectral mapping theorem it easily follows that the spectrum of an algebraic operator is a finite set. It is known that generalized Weyl's theorem is not generally transmitted to perturbation of operators satisfying generalized Weyl's theorem. In [13], they proved that if $T$ is paranormal and $F$ is an algebraic operator commuting with $T$, then Weyl's theorem holds for $T+F$. We now extend this result to generalized Weyl's theorem for algebraically quasi-paranormal operators. We begin with the following lemma.

Lemma 3.1. Let $T \in B(\mathcal{H})$. Then the following statements are equivalent:

(1) $T \in g \mathcal{W}$;

(2) $T$ has SVEP at every $\lambda \in \mathbb{C} \backslash \sigma_{B W}(T)$ and $\pi(T)=E(T)$.

Proof. Observe that $T \in g \mathcal{B}$ if and only if $\sigma_{B W}(T)=\sigma_{D}(T)$. So $T \in g \mathcal{B}$ if and only if $T$ has SVEP at every $\lambda \in \mathbb{C} \backslash \sigma_{B W}(T)$. Therefore we obtain the desired conclusion. $\square$

From this lemma, we obtain the following corollary

Corollary 3.2. Let $T \in B(\mathcal{H})$. Suppose $T$ has SVEP. Then

$T \in g \mathcal{W}$ if and only if $T \in \mathcal{P}_{1}(\mathcal{H})$.

Proof. Since $T$ has SVEP, $T \in g \mathcal{B}$ by Lemma 3.1. So $\sigma(T) \backslash \sigma_{B W}(T)=\pi(T)$. Therefore $T \in g \mathcal{W}$ if and only if $T \in \mathcal{P}_{1}(\mathcal{H})$ by Theorem 2.6.

Lemma 3.3. Suppose $T \in B(\mathcal{H})$ and $N$ is nilpotent such that $T N=N T$. Then $T \in \mathcal{P}_{1}(\mathcal{H})$ if and only if $T+N \in \mathcal{P}_{1}(\mathcal{H})$.

Proof. Suppose $N^{p}=0$ for some $p \in \mathbb{N}$. Observe that without any assumption on $T$ we have

$$
N(T) \subseteq N(T+N)^{p} \text { and } N(T+N) \subseteq N\left(T^{p}\right) .
$$

Suppose now that $T \in \mathcal{P}_{1}(\mathcal{H})$, or equivalently $\pi(T)=E(T)$. We show first $E(T)=E(T$ $+N)$. Let $\lambda \in E(T)$. Without loss of generality, we may assume that $\lambda=0$. From $\sigma(T$ $+N)=\sigma(T)$, we see that 0 is an isolated point of $\sigma(T+N)$. Since $0 \in E(T), \alpha(T)>0$ and hence by the first inclusion in (3.3.1) we have $\alpha(T+N)^{p}>0$. Therefore $\alpha(T+N)>0$, and hence $0 \in E(T+N)$. Thus the inclusion $E(T) \subseteq E(T+N)$ is proved. To show the opposite inclusion, assume that $0 \in E(T+N)$. Then 0 is an isolated point of $\sigma(T)$ because $\sigma(T+N)=\sigma(T)$. Since $\alpha(T+N)>0$, the second inclusion in (3.3.1) entails that $\alpha\left(T^{p}\right)$ $>0$. Therefore $\alpha(T)>0$, and hence $0 \in E(T)$. So the equality $E(T)=E(T+N)$ is proved. Suppose $T \in \mathcal{P}_{1}(\mathcal{H})$. Then $\pi(T)=E(T)$ by Theorem 2.6, and so $\pi(T+N)=\pi(T)=E$ $(T)=E(T+N)$. Therefore $T+N \in \mathcal{P}_{1}(\mathcal{H})$. Conversely, if $T+N \in \mathcal{P}_{1}(\mathcal{H})$ by symmetry we have $\pi(T)=\pi(T+N)=E(T+N)=E((T+N)-N)=E(T)$, so the proof is complete.

口

The following theorem is a generalization of [13, Theorem 2.5]. The proof of the following theorem is strongly inspired to that of it.

Theorem 3.4. Suppose $T$ is algebraically quasi-paranormal. If $F$ is algebraic with $T F$ $=F T$, then $T+F \in g \mathcal{W}$. 
Proof. Since $F$ is algebraic, $\sigma(F)$ is finite. Let $\sigma(F)=\left\{\mu_{1}, \mu_{2}, \ldots, \mu_{n}\right\}$. Denote by $P_{i}$ the spectral projection associated with $F$ and the spectral set $\left\{\mu_{i}\right\}$. Let $Y_{i}:=R\left(P_{i}\right)$ and $Z_{i}$ : $=N\left(P_{i}\right)$. Then $H=Y_{i} \oplus Z_{i}$ and the closed subspaces $Y_{i}$ and $Z_{i}$ are invariant under $T$ and $F$. Moreover, $\sigma\left(F \mid Y_{i}\right)=\left\{\mu_{i}\right\}$. Define $F_{i}:=F \mid Y_{i}$ and $T i:=T \mid Y_{i}$. Then clearly, the restrictions $T_{i}$ and $F_{i}$ commute for every $i=1,2, \ldots, n$ and

$$
\sigma(T+F)=\sigma\left((T+F) \mid Y_{i}\right) \cup \sigma\left((T+F) \mid Z_{i}\right) .
$$

Let $h$ be a nontrivial complex polynomial such that $h(F)=0$. Then $h\left(F_{i}\right)=h\left(F \mid Y_{i}\right)=$ $h(F) \mid Y_{i}=0$, and from $\{0\}=\sigma\left(h\left(F_{i}\right)\right)=h\left(\sigma\left(F_{i}\right)\right)=h\left(\left\{\mu_{i}\right\}\right)$, we obtain that $h\left(\mu_{i}\right)=0$. Write $h(\mu)=\left(\mu-\mu_{i}\right)^{m} g(\mu)$ with $g\left(\mu_{i}\right)=0$. Then $0=h\left(F_{i}\right)=\left(F-\mu_{i}\right)^{m} g\left(F_{i}\right)$, where $g\left(F_{i}\right)$ is invertible. Hence $N_{i}:=F_{i}-\mu_{i}$ are nilpotent for all $i=1,2, \ldots, n$. Observe that

$$
T_{i}+F_{i}=\left(T_{i}+\mu_{i}\right)+\left(F_{i}-\mu_{i}\right)=T_{i}+N_{i}+\mu_{i}
$$

Since $T_{i}+\mu_{i}$ is algebraically quasi-paranormal for all $i=1,2, \ldots, n, T_{i}+\mu_{i}$ has SVEP. Moreover, since $N_{i}$ is nilpotent with $T_{i} N_{i}=N_{i} T_{i}$, it follows from [6, Corollary 2.12] that $T_{i}+N_{i}+\mu_{i}$ has SVEP, and hence $T_{i}+F_{i}$ has SVEP. From [6, Theorem 2.9] we obtain that

$$
T+F=\bigoplus_{i=1}^{n}\left(T_{i}+F_{i}\right) \text { has SVEP. }
$$

Now, we show that $T+F \in \mathcal{P}_{1}(\mathcal{H})$. Since $T_{i}+\mu_{i}$ is algebraically quasi-paranormal, $T_{i}+\mu_{i} \in \mathcal{P}_{1}\left(Y_{i}\right)$ by Theorem 2.8. By Lemma 3.3 and (3.4.1), $T_{i}+F_{i} \in \mathcal{P}_{1}\left(Y_{i}\right)$ for every $i$ $=1,2, \ldots, n$. Now assume that $\lambda_{0} \in E(T+F)$. Fix $i \in \mathbb{N}$ such that $1 \leq i \leq n$. Since the equality $T_{i}+N_{i}-\lambda_{0}+\mu_{i}=T_{i}+F_{i}-\lambda_{0}$ holds, we consider two cases:

Case I: Suppose that $T_{i}-\lambda_{0}+\mu_{i}$ is invertible. Since $N_{i}$ is quasi-nilpotent commuting with $T_{i}-\lambda_{0}+\mu_{i}$, it is clear that $T_{i}+F_{i}-\lambda_{0}$ is also invertible. Hence $H_{0}\left(T_{i}+F_{i}-\lambda_{0}\right)=$ $N\left(T_{i}+F_{i}-\lambda_{0}\right)=\{0\}$.

Case II: Suppose that $T_{i}-\lambda_{0}+\mu_{i}$ is not invertible. Then $\lambda_{0}-\mu_{i} \in \sigma\left(T_{i}\right)$. We claim that $\lambda_{0} \in E\left(T_{i}+F_{i}\right)$. Note that $\lambda_{0} \in \sigma\left(T_{i}+\mu_{i}\right)=\sigma\left(T_{i}+F_{i}\right)$. Since $\sigma\left(T_{i}+F_{i}\right) \in \sigma(T+$ $F)$ and $\lambda_{0} \in$ iso $\sigma(T+F), \lambda_{0} \in$ iso $\sigma\left(T_{i}+N_{i}+\mu_{i}\right)$. Therefore $\lambda_{0}-\mu_{i} \in$ iso $\sigma\left(T_{i}+N_{i}\right)=$ iso $\sigma\left(T_{i}\right)$. Since $T_{i}-\lambda_{0}+\mu_{i}$ is algebraically quasi-paranormal, $\lambda_{0}-\mu_{i} \in \pi\left(T_{i}\right)$. Since $\pi\left(T_{i}\right)=E\left(T_{i}\right)$ by Theorem 2.6 and $T_{i} \in g \mathcal{W}$ by Theorem 2.9, $\lambda_{0}-\mu_{i} \in E\left(T_{i}\right)=\sigma\left(T_{i}\right)$ । $\sigma_{B W}\left(T_{i}\right)$. But $N_{i}$ is nilpotent with $T_{i} N_{i}=N_{i} T_{i}$, hence $\sigma_{D}\left(T_{i}\right)=\sigma_{D}\left(T_{i}+N_{i}\right)$ and $T_{i}+N_{i} \in g \mathcal{B}$. Therefore we have $\sigma_{B W}\left(T_{i}+N_{i}\right)=\sigma_{D}\left(T_{i}+N_{i}\right)$. Hence

$$
E\left(T_{i}\right)=\sigma\left(T_{i}\right) \backslash \sigma_{B W}\left(T_{i}\right)=\sigma\left(T_{i}+N_{i}\right) \backslash \sigma_{B W}\left(T_{i}+N_{i}\right) .
$$

Hence $T_{i}+F_{i}-\lambda_{0}$ is $B$-Weyl. Assume to the contrary that $T_{i}+F_{i}-\lambda_{0}$ is injective. Then $\beta\left(T_{i}+F_{i}-\lambda_{0}\right)=\alpha\left(T_{i}+F_{i}-\lambda_{0}\right)=0$. Therefore $T_{i}+F_{i}-\lambda_{0}$ is invertible, and so $\lambda_{0} \notin \sigma\left(T_{i}\right.$ $\left.+F_{i}\right)$. This is a contradiction. Hence $\lambda_{0} \in E\left(T_{i}+F_{i}\right)$. Since $T_{i}+F_{i} \in \mathcal{P}_{1}\left(Y_{i}\right)$ by Theorem 2.6, there exists a positive integer $m_{i}$ such that $H_{O}\left(T_{i}+F_{i}-\lambda_{0}\right)=N\left(T_{i}+F_{i}-\lambda_{0}\right)^{m i}$.

From Cases I and II we have

$$
\begin{aligned}
H_{0}\left(T+F-\lambda_{0}\right) & =\bigoplus_{i=1}^{n} H_{0}\left(T_{i}+F_{i}-\lambda_{0}\right) \\
& =\bigoplus_{i=1}^{n} N\left(T_{i}+F_{i}-\lambda_{0}\right)^{m_{i}} \\
& =N\left(T+F-\lambda_{0}\right)^{m},
\end{aligned}
$$


where $m:=\max \left\{m_{1}, m_{2}, \ldots, m_{n}\right\}$. Since the last equality holds for every $\lambda_{0} \in E(T+F)$, $T+F \in \mathcal{P}_{1}(\mathcal{H})$. Therefore $T+F \in g \mathcal{W}$ by Corollary 3.2.

It is well known that if for an operator $F \in B(\mathcal{H})$ there exists a natural number $n$ for which $F^{n}$ is finite-dimensional, then $F$ is algebraic.

Corollary 3.5. Suppose $T \in B(\mathcal{H})$ is algebraically quasi-paranormal and $F$ is an operator commuting with $T$ such that $F^{n}$ is a finite-dimensional operator for some $n \in \mathbb{N}$. Then $T+F \in g \mathcal{W}$.

\section{Acknowledgements}

The authors would like to express their thanks to the referee for several extremely valuable suggestions concerning the article.

\section{Authors' contributions}

All authors contributed equally to the writing of the present article. And they also read and approved the final manuscript.

\section{Competing interests}

The authors declare that they have no competing interests.

Received: 1 December 2011 Accepted: 17 April 2012 Published: 17 April 2012

\section{References}

1. Berkani, M, Sarih, M: On semi B-Fredholm operators. Glasgow Math J. 43, 457-465 (2001)

2. Berkani, M: On a class of quasi-Fredholm operators. Int Equ Oper Theory. 34, 244-249 (1999). doi:10.1007/BF01236475

3. Berkani, M, Koliha, Jj: Weyl type theorems for bounded linear operators. Acta Sci Math (Szeged). 69, 359-376 (2003)

4. Han, YM, Na, WH: A note on quasi-paranormal operator. Mediterr J Math. (in press)

5. Curto, RE, Han, YM: Generalized Browder's and Weyl's theorems for Banach space operators. J Math Anal Appl. 336, 1424-1442 (2007). doi:10.1016/j.jmaa.2007.03.060

6. Aiena, P: Fredholm and Local Spectral Theory, with Applications to Multipliers. Kluwer Academic Publishers, Dordrecht (2004)

7. Curto, RE, Han, YM: Weyl's theorem holds for algebraically paranormal operators. Int Equ Oper Theory. 47, 307-314 (2003). doi:10.1007/s00020-002-1164-1

8. Han, JK, Lee, HY, Lee, WY., et al: Invertible completions of $2 \times 2$ upper triangular operator matrices. Proc Am Math Soc. 128, 119-123 (2000). doi:10.1090/50002-9939-99-04965-5

9. Berkani, M: Index of B-Fredholm operators and generalization of a Weyl theorem. Proc Am Math Soc. 130, 1717-1723 (2002). doi:10.1090/S0002-9939-01-06291-8

10. Aiena, P, Garcia, O: Generalized Browder's theorem and SVEP. Mediterr J Math. 4, 215-228 (2007). doi:10.1007/s00009007-0113-2

11. Laursen, KB, Neumann, MM: An Introduction to Local Spectral Theory. London Mathematical Society Monographs New Series 20, Clarendon Press, Oxford (2000)

12. Aiena, P, Miller, TL: On generalized a-Browder's theorem. Studia Math. 180, 285-300 (2007). doi:10.4064/sm180-3-7

13. Aiena, P, Guillen, JR: Weyl's theorem for perturbations of paranormal operators. Proc Am Math Soc. 135, 2443-2451 (2007). doi:10.1090/50002-9939-07-08582-6

\section{Submit your manuscript to a SpringerOpen ${ }^{\circ}$ journal and benefit from:}

- Convenient online submission

- Rigorous peer review

- Immediate publication on acceptance

- Open access: articles freely available online

- High visibility within the field

- Retaining the copyright to your article

Submit your next manuscript at $\$$ springeropen.com 